\title{
DECISION SUPPORT TOOLS FOR DOMESTIC SECURITY OPERATIONS
}

\author{
Yvan GAUTHIER and Jay ADAMSSON
}

\begin{abstract}
This paper documents the production of a compendium of modelling and simulation (M\&S) tools useful for decision support to organizations engaged in domestic defence and security operations. The compendium was created on GCPEDIA, a wiki application accessible to all federal government departments and agencies in Canada. It includes tools for predicting the consequences of natural and man-made disasters, planning major event security, assessing infrastructure criticality, planning surveillance missions, and analyzing other issues relevant to domestic operations. Given the wide accessibility of the compendium within the Government of Canada, a large community of practice can use, update, and expand on the information collected.
\end{abstract}

Keywords: modelling, simulation, decision support, domestic operations, civil assistance, natural disasters, major security events, CBRNE, critical infrastructure, pandemics, wiki.

\section{Introduction}

\section{Background}

Canada Command (Canada COM) is the Canadian Forces (CF) authority responsible for the conduct of all domestic operations in Canada, both routine and contingency, less those operations executed under the direct command of the Chief of Defence Staff or the North American Aerospace Defense Command (NORAD). Supported by six Regional Joint Task Forces (RJTFs), Canada COM continuously monitors developments across North America and tracks potential threats to Canada, from threatening natural phenomena such as hurricanes and floods, to security threats such as smuggling and terrorism. ${ }^{1}$

Canada COM offers a single point of contact for the many safety, security and defence partners of the CF. When requested, it coordinates military assistance to civil authorities, including consequence management. The command is also responsible for military support to the security of major events, such as the recent Vancouver 2010 
Olympic and Paralympic Winter Games (V2010) and the G8/G20 Leaders Summits held in Ontario in June 2010.

Operational analysts from Defence R\&D Canada - Centre for Operational Research and Analysis (DRDC CORA) are embedded within Canada COM. They provide analytical support to the Commander and subordinate RJTF commanders for improving the efficiency and effectiveness of domestic operations. The warning time for these operations may be substantial, for instance in planning military support to V2010 security, or very short for contingency operations such as disaster response. Accordingly, the time available for identifying, developing, and using modelling and simulation (M\&S) tools that meet decision makers' analysis requirements may vary significantly from one situation to another.

\section{Issue}

Domestic operations may be time-critical and are often, because of their proximity and impact on local populations, more publicly sensitive than international operations. It is thus essential for any decision support to military and civil authorities to be relevant and responsive to short-notice requirements. However, the M\&S tools that have been employed by the defence science community over the years have been very much oriented toward traditional expeditionary operations. They may not be adequate or sufficient to analyze the entire range of domestic threats and operations, especially operations in support of civil authorities.

As a first step to address these deficiencies, we assembled a compendium of M\&S tools available from government, commercial, or academic sources. ${ }^{2}$ The goal was to provide Canada COM, RJTFs, and their civilian security partners with an overview of existing M\&S tools, their capabilities, requirements, and points of contact. In addition, the compendium would allow Canada COM's operational analysts to identify relevant tools more quickly when needed, permitting a faster response to the Commander's questions and analysis requirements. Assembling this compendium required gathering information on a wide array of tools and producing an overview of each of them. It also required compiling the information in such a way that it would be easy to access, maintain, and update by all the stakeholders.

\section{Aim and outline}

The aim of this paper is to present how the compendium was produced and to give some examples of M\&S tools that are (or could be) useful for decision support to military and civilian organizations involved in domestic security operations. 
Following this introduction, we first describe the methodology and information sources that we used to produce the M\&S tool compendium. Then we summarize some of the tools that have been documented to date. We also highlight gaps in M\&S capabilities that may offer some opportunities for the future development of models, simulations, or analysis techniques.

\section{Compendium production}

\section{$M \& S$ tools of interest}

At the onset of the project, different categories of tools potentially useful to Canada COM and civilian partners were identified. They were based on a review of the command's concept of operations, the Commander's priorities, and meetings with senior staff from the plans (J5) and operations (J3) sections. The scope of tools of interest was also influenced by actual events that Canada COM had to prepare for during the course of the project, such as V2010 and the H1N1 pandemic.

The following types of decision support tools were prioritized:

- Tools for predicting the potential consequences of natural disasters such as storms, hurricanes, floods, earthquakes, tsunamis, forest fires, or other disasters.

- Tools for predicting the potential consequences of man-made disasters such as Chemical, Biological, Radiological, Nuclear and High Explosives (CBRNE) attacks or non-intentional releases.

- Tools for assessing the relative importance of critical infrastructures, their interdependencies, and the possible consequences of their failures.

- Tools for predicting the potential consequences of pandemics and preparing response plans.

- Tools for planning major event security, including tools that can model the behaviour of large crowds in urban environments.

- $\quad$ Other tools relevant in a domestic context, for instance tools for modelling intelligence, surveillance, and reconnaissance (ISR) activities or assessing force protection measures.

No discrimination was made between operations for which military forces are expected to play a lead role, and those for which they would be supporting civilian organizations. Tools specifically designed for the analysis of force generation or force development issues were out of scope, mainly because of the force employment role of Canada COM, but also because many of these tools had already been inventoried in previous studies. ${ }^{3,4,5,6}$ 


\section{Tool characteristics of interest}

Hundreds, if not thousands, of M\&S tools fall under the categories listed above. Hence a certain balance between the number of tools documented and the level of detail provided for each of them had to be found. Many key characteristics were documented, but no in-depth review, verification, or validation of the tools were conducted. Nonetheless, the tool descriptions refer to validation work previously carried out and examples of recent usage for decision support.

Specifically, the following pieces of information were captured for each tool, whenever available:

- Synopsis: a very brief description of the tool, in two or three lines.

- Description: a more detailed overview of the tool, including a high-level description of the mathematical techniques and logical processes applied.

- Level of application: a sentence describing if the tool is most useful in support of tactical, operational, or strategic decision making.

- Output: the main pieces of information that can be obtained by using the tool and the format in which they are produced.

- Examples of usage: real-world situations for which the tool has been used.

- Requirements:

- Input data: the data required to run the program;

- Platform: the computer hardware required;

- Software: the exploitation system and additional software packages required;

- Expertise: the knowledge and competencies required to gather the input data, run the tool, and interpret the output;

- Runtime: an order of magnitude approximation of the time required for a typical scenario to run. This does not include the time required to gather and enter the input data, which generally depends on the problem at hand;

- Additional resources: any other requirements not listed.

- Data sources: existing data sources or repositories for running the tool.

- Validation status and method: information on how well M\&S results reflect real-world phenomena or processes, and the method by which this was assessed.

- Strengths and weaknesses: the main advantages and disadvantages in selecting the tool for particular applications. 
- Support/user community: any area where assistance on using the tool can be obtained.

- Points of contact:

- Custodian: the person or organization that controls the structure and maintenance of the tool;

- Release authority: the person or organization that controls the release and use of the tool;

- $\quad$ References: any webpage, report, or manual about the tool.

\section{Information collection}

Collecting this information required casting a broad net inside and outside DRDC. Web searches proved to be the most fruitful method of collecting information. Leading tools in their fields are generally mature, have been in use for extended periods, and have established constituencies. This naturally leads to a strong web presence.

Interviews with scientists from DRDC, the U.S. Northern Command (USNORTHCOM), and other organizations were also conducted. In many cases, they pointed to other tools, points of contact, or scientific reports describing various operationally-relevant tools and case studies.

Scientific journals were also surveyed, but this approach was not as fruitful. Articles published in journals generally described early-stage research, or one-off modelling approaches to problems. A few articles led to more fully-developed tools, but they generally replicated information already gathered through other approaches.

\section{Documentation}

A lot of consideration was given to the support on which the information would be compiled and organized. To maximize the long-term utility of the compendium, it had to be easy to access, maintain, search, and update.

We considered different support options and rejected the idea of compiling the information in report form only. In the past, similar tool collections that were documented in reports have been most useful, but even those that aimed to remain living documents were hardly ever updated after their original publication. Unless the compendium could be accessed and updated by a large community of analysts, it would probably end up in the same way. For this reason, a collaborative web-based environment was a much preferable option. 
Two types of collaborative environments were considered: web portals (e.g., Microsoft SharePoint portals) and wikis. We selected the second option because wikis are more appropriate for collaborating on formalized, text-based descriptions. They provide an open approach to information as anyone can update it or add to it. Moreover, wikis are easy to search and generally keep track of modifications previously made, a valuable feature for writing technical documentation collaboratively. They are also increasingly easy to use, as proven by the 91,000 active contributors to Wikipedia worldwide. $^{7}$

\section{GCPEDIA}

We identified GCPEDIA as the most promising platform to host the compendium. GCPEDIA is a proof-of-concept wiki stood up by Canada's Treasury Board Secretariat for a common, easily accessible collaborative workplace for the entire Government of Canada's public service. ${ }^{8}$ GCPEDIA runs on the MediaWiki software, the same open-source software that runs Wikipedia, and is accessible from most federal government networks. From approximately 5,000 registered users we started to document M\&S tools in June 2009, GCPEDIA has now over 22,000 registered users across all federal departments and agencies and this number still grows rapidly.

Figure 1 shows an example of a M\&S tool that was documented in GCPEDIA.

\section{Examples of relevant M\&S tools}

The following pages present synopses of some of the tools that have been documented in the compendium. They are presented by category. Tools that fit multiple categories (e.g., disaster models that may help to predict critical infrastructure damage) are presented only once in the category that suits them most. In GCPEDIA however, tools that are relevant to multiple categories appear in all of them since wikis do not have a hierarchical page structure.

\section{Natural and man-made disasters}

Table 1 presents examples of M\&S tools relevant to various types of natural and man-made disasters, including hurricanes, earthquakes, floods, fires, tsunamis, and CBRNE incidents. The main purpose of these tools is to estimate disaster consequences such as casualties, evacuation requirements, infrastructure damage, shelter availability, and other information of interest. They can also be used for performing analyses in support of operational planning and contingency planning. 


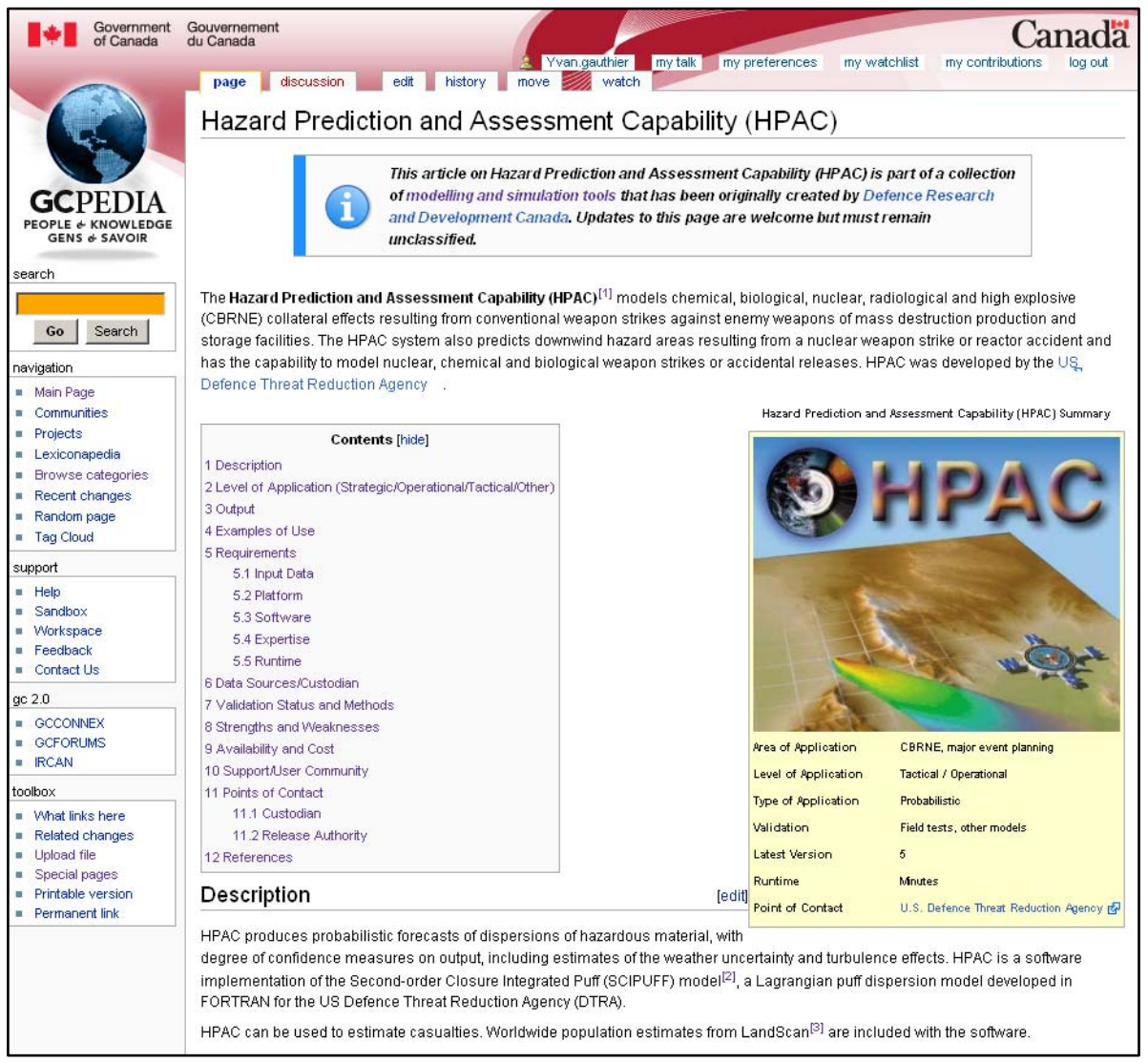

Figure 1: Screenshot of a tool description on GCPEDIA.

\section{Gaps}

None of the tools directly models the consequences of snow storms or ice storms, which have led to the largest domestic CF operation to date (OP RECUPERATION in 1998). Nor can any of the identified tools model oil spill response operations, which may require military assistance if they were to occur in remote areas such as the Arctic.

Furthermore, although they can help predict disaster consequences, none of the tools directly models the military response to disaster scenarios. In order to better inform consequence management plans, it would be useful to find or develop tools that can derive more directly military requirements from the predicted disaster consequences. 
Table 1: Examples of M\&S tools for natural and man-made disasters.

Tool

Hazard U.S.

Multi-Hazard

(HAZUS-MH)

\section{Synopsis}

Developed and used by the U.S. Federal Emergency Management Agency (FEMA), HAZUS-MH is a simulation tool suite for predicting the damage from natural disasters, including floods, earthquakes, and hurricanes. Assists government officials in evaluating, planning for, and mitigating the effects of disasters, and making wise use of emergency management resources.

Hurricane
Planning Tracker

Developed and used by USNORTHCOM to assist the command in hurricane response planning. Models the strength and size of a hurricane, and estimates damage from landfall.

FloodArea

Commercially-developed extension of ArcGIS that simulates inundation during a flood event and displays its evolution over time.

Method of

Developed and used by the U.S. National Oceanic and Atmospheric

Splitting Tsunami

(MOST) Administration (NOAA) for predicting, given an earthquake, a tsunami's wave heights and propagation.

BehavePlus

Developed by the Missoula Fire Sciences Laboratory and used by the U.S. Forest Service, BehavePlus is part of a suite of fire behaviour systems that includes FARSITE (presented below). It is the simplest tool of the suite and describes the behaviour of forest fires and their effects for a set of uniform terrain and weather conditions.

Fire Area

Simulator

(FARSITE)
Computes wildfire growth and behaviour for long time periods under heterogeneous conditions of terrain, fuels, and weather. Designed for use by trained wildland fire planners.
Hazard Prediction and Assessment Capability
Developed by the U.S. Defence Threat Reduction Agency for modelling CBRNE collateral effects. Predicts downwind hazard areas resulting from a CBRNE weapon strike or accidental releases.
CBRNE

Response
Commercial software that provides CBRNE incident commanders on the ground with information for assisting with the operational tasking of both military and civil emergency agencies. 


\section{Critical infrastructures}

Protecting critical infrastructures (CIs) has become a priority for government departments and agencies over the last decade. Table 2 describes four CI tools that have all been developed, at least partially, under government sponsorship and funding. All of them can help planners to understand the interdependence of different CI sectors, and their relative priorities in terms of CI protection requirements.

The first two models (Critical Infrastructure Preventive Approach and I2Sim) are still undergoing active development, but have been used in recent studies such as CI vulnerability studies done prior to V2010. The approach implemented in RiskOutlook was originally developed by defence scientists for OP ABACUS (CF preparation for the $\mathrm{Y} 2 \mathrm{~K}$ bug) and has since evolved to become a commercial software. N-ABLE concentrates on the economic implications of CI failures.

Specific CI sectors (e.g. electricity grids, water transportation systems) have been the subject of extensive modelling efforts by academia and government agencies. Because of the sheer number CI-related tools available, the focus remained on generic tools that are broadly applicable and could be used to analyze how different CI entities or sectors relate to each other. An inventory of sector-specific CI models and simulations produced by the U.S. Department of Homeland Security ${ }^{9}$ lists several other models for tackling questions related to particular CI sectors.

Table 2: Examples of M\&S tools for CIs.

Tool

Synopsis

Critical

Infrastructure

Preventive

Approach

\section{I2Sim}

Developed by the Ecole Polytechnique de Montréal, this modelling framework has been implemented in different analysis tools. It models CIs and focuses on the knock-on impact of their failures on other CI sectors.

The Infrastructure Interdependencies Simulator (I2Sim) was developed by the University of British Columbia (UBC). It is an interdisciplinary model that simulates the extensive human and physical infrastructure interdependencies that need to be understood and coordinated for coping with disaster scenarios.
RiskOutlook
Commercial software that helps identify and prioritize risks among CIs. It also helps assess the interdependencies between pairs of in- frastructure components.

N-ABLE

Developed by Sandia National Laboratories, N-ABLE is an agentbased simulation of an economy. It is used to determine the vulnerability of CIs and assess economic security issues. 
Gaps

It must be stressed that CI modelling is often very challenging, partly because of the number of entities involved, but also because of privacy and confidentiality issues surrounding CI data. Disparate data sources, the lack of data standards, and the dynamic environment of some CI sectors also contribute to make data collection the most important gap in CI modelling. Hence, even the simplest models might be difficult to feed with appropriate data. Canada COM is currently developing its own infrastructure database, which will alleviate some of these data collection issues.

\section{Pandemics}

The recent H1N1 influenza pandemic highlighted the need for models that can help reduce uncertainty and inform decisions makers during the preparation and execution of response plans. Some M\&S tools relevant to pandemics are shown in Table 3.

Certain tools, such as EpiCast, EpiSimS, and CommunityFlu are concerned with the spread of a pandemic, either across an entire country's population, an urban area, or a small community. BERM, Panalysis, and RealOpt can be used for analyzing prophylactic dispensing and health services logistics.

The tools vary from simple and easy-to-use spreadsheet models, to sophisticated agent-based models that can simulate several millions of people. Since the military might be asked to assist civil authorities during a pandemic, the tools could be used for different purposes. For instance, they can be used in conjunction with some of the CI M\&S tools previously presented to help predict what CIs or services would be the most affected, and thus forecast where military support might be required.

Modelling can also help predict how a pandemic would affect military and civil forces at different locations. However, the transmission of a virus throughout a population, whether on a national scale or community scale, is dependent upon several factors (virulence, population mobility and interaction patterns, mitigation measures in place, etc.). Large uncertainties surround these factors, and it is often difficult to produce precise estimates of casualties and attack rates. As such, virus transmission models such as EpiCast are best used for comparing different mitigation approaches, or for determining worst-case scenarios, rather than making authoritative predictions on the virus spread and its impact.

Logistic models such as BERM and RealOpt would probably be of a greater utility to operational commanders. They can help develop vaccination plans that can be executed quickly under various resource constraints and situations. For instance, BERM considers the time required for travelling between locations and setting up vaccination clinics. This travel time is an important factor to consider when providing assis- 
tance to health authorities in remote communities, something military forces might be requested to do.

Table 3: Examples of M\&S tools for pandemics.

\begin{tabular}{ll}
\hline Tool & \multicolumn{1}{c}{ Synopsis } \\
\hline EpiCast & $\begin{array}{l}\text { Developed by the Los Alamos National Laboratory (LANL), Epi- } \\
\text { Cast models the behaviours of a population on a national scale and } \\
\text { predicts how a pandemic will spread. Various mitigation measures } \\
\text { can be simulated to compare their benefits. }\end{array}$
\end{tabular}

Epidemiology

System

(EpiSimS)
Also developed by LANL for simulating the spread of epidemics in large urban regions, taking into account realistic contact patterns and disease transmission characteristics. It uses industry data for estimating the economic impact due to absenteeism and reallocation of resources.

CommunityFlu

Developed by the U.S. Centers for Disease Control (CDC) for modelling the spread of influenza through a community of approximately 2500 residents. It estimates number of deaths, hospitalizations, and outpatient visits. Possible interventions can be modelled, allowing the comparison of various response strategies.

Panalysis

Commercial spreadsheet tool that models the impact of resources, or shortage thereof, on health services during a pandemic. Shows the impact of bed and space augmentation, early discharge of patients, cancellation of elective surgeries, fatalities due to ventilator and bed shortages, as well as staff requirements.

RealOpt

Developed by GeorgiaTech for assisting authorities in creating and testing more efficient plans for emergency response logistics and treatment of infectious illness, whether it is a natural or man-made outbreak. Has been used for the planning of Anthrax emergency exercises and actual flu vaccinations.

BERM

Developed by the U.S. Department of Health, the Bioterrorism and Epidemic Outbreak Response Model (BERM) model predicts the number and type of staff needed to respond to a major disease outbreak or bioterrorism attack on a given population. 


\section{Gaps}

Although many tools can inform the preparation of pandemic response plans, there is no ideal tool for assessing and mitigating the spread of a pandemic on forces personnel themselves. But it would be feasible to adapt a tool such as CommunityFlu to estimate virus spread within particular military communities or military bases. Furthermore, none of the documented tools can directly assess the impact of a pandemic on force readiness based on the fraction of staff affected. It is unclear if such a tool exists, but some existing readiness models or business continuity models could possibly be modified to estimate a pandemic's impact on military readiness.

\section{Major event security}

Military forces often provide support to civilian authorities in planning and providing security for major international events. Table 4 presents M\&S tools that are useful for planning specific security aspects, such as vehicle screening (VISTA-VSA) or personnel shift scheduling (Shift Scheduler). Both tools have been recently developed by DRDC CORA and used in support of V2010 security planning. The data required by these tools depend on the particulars of the event, but the tools themselves could be re-used for future major events with little or no modifications.

Various crowd modelling tools are also documented in the compendium, such as EXODUS, STEPS, and Legion. These are well-established commercial tools that can be used for planning evacuations of venues, or for simulating pedestrian activity within urban operations models. Each tool employs a slightly different approach and has its own strengths and limitations. Some have already been used for specific types of venue (e.g., stadia, subway stations) or specific types of problem (e.g., evacuation planning, emergency response, crowd management). Finding which tool is the most appropriate depends on the problem at hand. Generally, input data requirements for crowd modelling tools are not too demanding, but venue plans and/or terrain characteristics must be entered into the model.

Gaps

Much has been learned from the analysis work done for the CF and their civilian partners prior to V2010 and the G8/G20 Leaders Summits. Many of the tools that have been used in preparation for these events have been included in the compendium. But more tools could be added, for instance those that were used for command and control (C2) studies and the design of the security operation centres. These tools will be added to the compendium by another DRDC team aiming to develop a major event security framework. 
Table 4: Examples of M\&S tools for major event security planning

\section{Tool}

Synopsis

Shift Scheduler

Developed by DRDC CORA for the V2010 Integrated Security Unit (ISU). Determines shift schedules that minimize operational costs over a fixed time period.

VISTA-VSA

The Vancouver Integrated Screening Team Assessment - Vehicle Screening Area (VISTA-VSA) model was also developed by DRDC CORA for the V2010 ISU. It simulates personnel, resources and physical areas where vehicle screening occurs, with the objective of determining wait times for different resource levels and screening area configurations.

EXODUS

Developed by the University of Greenwich to simulate crowds and their evacuation from a variety of venues under normal and emergency circumstances.

Legion

Commercial, agent-based simulation that models pedestrian activity. Can be used for evacuation studies or for simulating other emergency scenarios occurring in urban environments.

STEPS

The Simulation of Transient Evacuation and Pedestrian MovementS (STEPS) is a commercial software that simulates the movement of people under both normal and emergency conditions in various locations such as office buildings, shopping centres, sports stadia, subway, and train stations

\section{Conclusion}

At time of writing, $50 \mathrm{M} \& \mathrm{~S}$ tools for planning and analyzing different aspects of domestic security had been documented in the compendium. Most of them are operationally relevant and cover domestic issues for which military forces and civil authorities may require decision support.

Surely, these tools represent only a small subset of all the models and simulations available from government, academic, or commercial sources. Nonetheless, this effort should be seen as first step toward a larger tool compendium, which has the advantage of being built on a collaborative and expandable platform. Since GCPEDIA is accessible to all federal departments and agencies, a large community can revise, update, or expand on the information collected. The M\&S tool compendium is already a 
central element of a Major Events Security Framework (MESF) currently being developed in Canada. It will be a re-useable, collaborative framework for helping police forces, the $\mathrm{CF}$, and their mission partners in planning major event security. The MESF will build upon the lessons learned, tools, and studies produced from the V2010 and G8/G20 Leaders Summits experience.

The M\&S gaps described in this paper offer many opportunities for further research and development. One key area of research would be to determine joint force requirements based on the consequences of different types of events, in order to improve risk analysis and modify the posture of response units when necessary.

\section{Notes:}

1. Department of National Defence, Canada Command Backgrounder (2010), www.canadacom.forces.gc.ca/nr-sp/bg-do/10-001-eng.asp (26 Feb. 2011).

2. Yvan Gauthier and Jay Adamsson, "Modelling and Simulation Tools for Decision Support to Canada Command,” DRDC CORA Technical Report 2010-177 (2010).

3. E.J. Emond, D.G. Hunter, I.W. Taylor, T. Yazbeck, and P.L. Massel, "Maritime Operational Research Tool Set Review - Final Report,” DRDC CORA Technical Memorandum TM2005-28 (2005).

4. E.J. Emond and D.W. Mason, "Report on the Review of Permanent Model and Simulation Requirements to Support Studies of CF Human Resources Issues," DRDC CORA Research Note RN2000/12 (2000).

5. D.W. Mason and E.J. Emond. "Review of Land Force Operational Research Tool Final Report,” DRDC CORA Project Report PR2003-13 (2003).

6. N. Curtis, J.R. Field, D.S. Haslip, and P.J. O’Neill, “TTCP Technical Report TR-LND- AG1-3-2009,” TTCP Action Group 1 (AG-1) (2009).

7. About Wikipedia, http://en.wikipedia.org/wiki/Help:About (21 Feb. 2011).

8. “Government creates own version of Wikipedia," Ottawa Citizen, 29 October 2008, www.canada.com/ottawacitizen/news/story.html?id=3fcb24f9-22c9-4e918656-2a252c1e8cd4 (21 Feb. 2011). 
9. N. Adam, Workshop on Future Directions in Critical Infrastructure Modeling and Simulation - Final Report (Washington, D.C.: Department of Homeland Security, 2008).

Yvan GAUTHIER is a Defence Scientist with Defence R\&D Canada - Centre for Operational Research and Analysis (DRDC CORA). Since 2008, he leads a team of operational research analysts embedded with Canada Command, the Canadian Forces (CF) organization responsible for CF operations in Canada and North America, including operations in assistance to civil authorities. Mr. Gauthier began his career with DRDC CORA in 2000, first working for the Chief of the Air Staff organization and then for Joint Task Force Pacific, the operational command responsible for all CF operations on Canada's West Coast. He has also worked with Dstl Naval Systems in Porstdown West, UK as an exchange scientist from 2006 to 2008. Mr. Gauthier has a Masters degree in Physics from Carleton University, Ontario, Canada.

E-mail: yvan.gauthier@drdc-rddc.gc.ca.

Jay ADAMSSON is a private consultant specializing in operational research, business analytics, and web analytics. He is the owner of Analytic-OR, a consulting and business services company based in Prince Edward Island. Before founding AnalyticOR, he spent over ten years working for DRDC CORA. During this time, Dr. Adamsson was responsible for the determination and implementation of a data collection and analysis system to support large purchase decisions, and the determination of the fleet sizes required by the Air Force to meet their stated missions. He is a specialist in the application of mathematical processes and techniques to real-world problems. Dr. Adamsson has also worked as a cryptographer and as an assistant professor at the University of Prince Edward Island. He obtained his Doctorate in Science (Mathematics) from Carleton University, Ontario, Canada in 2000.

E-mail: jay@analytic-or.com. 